\title{
Cloud Computing Technique Applied to Detect Cyber-Attacks on Web Application
}

\author{
Babeetha Muruganantham, Akash Modi, Eshwesh Gehlot, Abhishek Botikar
}

\begin{abstract}
The targeted malignant emails (TME) for PC arrange misuse have become progressively deceptive and all the more generally common as of late. Aside from spam or phishing which is intended to fool clients into uncovering individual data, TME can misuse PC systems and accumulate touchy data which can be a major issue for the association. They can comprise of facilitated and industrious battles that can be terrible. Another email-separating procedure which depends on bowl classifier and beneficiary arranged highlights with an arbitrary backwoods classifier which performs superior to two conventional recognition techniques, Spam Assassin and Clam AV, while keeping up sensible bogus positive rates. This proposed model deals with how to recognize a pernicious bundle (email) for ordinary system into current system. We build up an undermined protocol of network detection that powerfully concludes the correct number of congestive loss of packets that is going to happen. On the chance that one damages the steering convention itself, at that point aggressor may make enormous segments of the system become untreatable. We build up an option shifting technique by utilizing TME explicit element extraction. Our conventions naturally anticipate clog in a deliberate manner, as it is vital in making any such flaw in network recognition reasonable.
\end{abstract}

Keyword: TME, Random forest classifier, Waterfall model, $V$-model

\section{INTRODUCTION}

In today's world social media is used by myriad of people and due to this cyber-crime has increased a lot. As new social media applications are coming day by day, emails are now less preferred for chatting and has taken a professional seat. Emails are now preferred by schools, colleges and other business areas where any professional work is needed. According to the result of the recent survey, nearly 3.9 billion uses email, which is $50 \%$ of population of the world. And among those people, around 55\% of the emails are spam messages. It accounts for 14.5 billion emails per day worldwide. As a result it is very important to filter spam emails. There are nowadays many malignant emails which are sent as a mail or an attachment through mails to various targeted computer networks.

Revised Manuscript Received on April 18, 2020.

* Correspondence Author

Mrs. Babeetha Muruganantham*, Dept. of Information Technology, SRM IST, Chennai, India babeethas14@gmail.com

Mr. Akash Modi, Dept. of Information Technology SRM IST, Chennai, India akashmodi.31198@gmail.com

Mr. Eshwesh Gehlot, Dept. of Information Technology SRM IST, Chennai, India eshweshgehlot@gmail.com

Mr. Abhishek Botikar, Dept. of Information Technology, SRM IST, Chennai, India botikarabhishek@gmail.com

(c) The Authors. Published by Blue Eyes Intelligence Engineering and Sciences Publication (BEIESP). This is an open access article under the CC BY-NC-ND license (http://creativecommons.org/licenses/by-nc-nd/4.0/)
Once the user opens the mail or clicks on There are nowadays many malignant emails which are sent as a mail or an attachment through mails to various targeted computer networks. Once the user opens the mail or clicks on the attachment, the virus slowly starts its process in destroying the computer network. It might also aim to get the information of the organization which is hidden and could also leak the information. There is an existing system that deals with the malicious emails but the system uses protocols which are distributed to detect the manipulations of traffic. The attacker can easily subvert the plane of network control by manipulating the router protocol with another route. Hence the new system is proposed which follows an undermined protocol for the detection of router that is dynamic and it gathers the amount of packet losses in traffic which are going to happen. When the routing protocol is damaged by its own, the enormous segments of the system can be made untreatable by the attacker. Consequently, an option shifting method is developed by utilizing TME explicit element extraction. The protocols instinctively anticipated congestion in an orderly way and it is important in making such detection of network fault practical.

\section{RELATED WORKS}

\section{A. SPD}

Proposed a successful structure for identification spam in twitter called it as SPD. They previously used an improved arrangement of highlights autonomous of recorded tweets, and afterward applied a lot of AI techniques, for example, irregular timberland, multilayer perceptron (MLP), bolster vector machine (SVM), and "additional trees" on both Honeypot [17] and an elite subset of Honeypot dataset to show the viability and heartiness of the proposed strategy. The outcomes displayed that the SPD chose datasets can give better outcomes in term of exactness and F-score in the greater part of the classifiers; in any case, there are a few classifiers like "additional trees" that increase lower F-score and recal in the SPD chose dataset.

\section{B.Semi-Supervised Spam Detection}

S3D structure for spam discovery in Twitter uses four light weight identifiers to identify spam tweets in a continuous way and update some learning models intermittently for each piece of information. They utilized Naïve Bayes other than of irregular woods and calculated relapse for grouping approaching information, and afterward they tried their model more than 15 days of HSpam14 dataset [19].

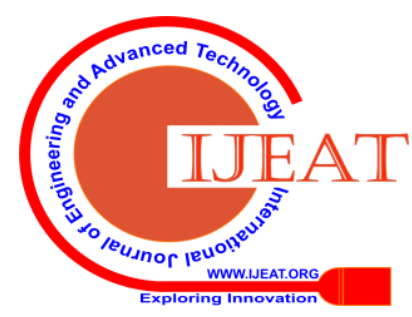


They guaranteed that the proposed S3D was more powerful than the completely directed methods for catching spam tweets. S3D gave better precision value contrasted to that of random forest over three days of streaming data.

\section{PROPOSED MODEL}

A noxious email message is one which have been intentionally made to cause issues on the server or on the customer. This could be because of the message containing an infection, or it could be expected to that message being created so as to exploit a shortcoming in the getting mail customer. GMS gives a scope of checks which might be run against all messages going through the framework to keep this sort of message from entering the server by any means. On the off chance that you would prefer not to boycott the messages altogether it will add an admonition to the approaching message. These checks are known as Message Quality checks, and investigate the substance of each message to guarantee it is fundamentally stable, just as searching for structures that are regularly intended to take advantage of defects in a portion of the more famous mail customers.

1. An undermined protocol of router detection which is dynamic, gathers the amount of packet losses in traffic which are going to happen.

2. By disregarding the directing convention itself, an aggressor may cause huge parts of the system to get inoperable.

3. We create an alternative separating system by utilizing TME explicit element extraction.

4. Our conventions naturally foresee clog in a methodical manner.

5. It is essential to make such shortcoming of network recognition viable.

6. A rearranged perspective on our order procedure initially includes preprocessing email, utilizing organization explicit data. Tenacious danger and beneficiary oriented features are separated and the related messages are arranged utilizing an irregular woodland classifier.

7. To break down and locate the pernicious email (bundle) by utilizing highlight extraction strategy.

8. The dataset used to assess email-sifting methods of a few diverse datasets. Datasets comprise of three classes of email. For example,

- Directed Malicious Email (TME).

- Non-Targeted Malicious Email (NTME).

- Assessment set containing both TME and NTME.

9. We utilized NTME and TME to build the TME-channel system and give setting to the new highlights we joined for TME recognition.

\section{METHODOLOGY \& IMPLEMENTATION}

\section{a. Waterfall Model}

The Waterfall Model functions admirably when the product prerequisites are surely known and the idea of the product advancement includes legally binding understandings. The model is a breakdown of the project in linear sequence where in each stage depends on the activity of the previous one and corresponds it to specializations of tasks. This model is used in the design of particular structures in engineering and software development. It is the most flexible model and follows less iterative approaches. It does move ahead in multiple directions, it goes in singular direction which is linear. It follows series of steps which starts with formation of the entire plan called conception, then the next step is to initiate the plan which is known as initiation. Furthermore, one needs to construct and design the whole idea. The moment it is done analysis is done to figure out the errors. Later on after solving the mistakes, a proper model is made and its maintenance should be done accurately.

This model uses one of the least demanding and complex strategy in comparison to other models. It makes easier for the business perspective to use it and solve the problems. Anyone can comprehend the model and move ahead by following simple steps. When the stage of testing comes, it becomes difficult for people to resolve the issues. Hence it requires sort of more work. But after that one needs to integrate the progress into a single structure and framework.

\section{b. V-Model}

This model is also known as U-Model. It mirrors the way to deal with frameworks improvement where in the definition side of the model is connected legitimately to the affirmation side. It indicates early testing and arrangement of testing situations and cases before the construct stage to at the same time approve the definitions and get ready for the test stages. The model is the state of the advancement cycle and the idea of it down and over the stages. The model shows the normal arrangement of advancement exercises on the left-hand (downhill) side and the relating grouping of test execution exercises on the right-hand (tough) side. It gives user a deal of proper alignment of specification and testing. This is additionally beneficial to the people in business who want to utilize the model and also imply consideration firstly of testing which is done on a later stage.

In the phase of implementation, from the start, we utilized a ten fold cross approval as our assessment strategy for the joint dataset which is NTME1-TME1. Afterward, the same joint dataset is utilized for further training process, however in place of repeating the same process to access the targeted malicious email channel developed utilizing the same joint dataset, we utilized free TS1 dataset to do the process.

Once an email is received through the use of feature extraction using random forests, classification is done on the basis of non targeted malicious emails and targeted malicious emails.

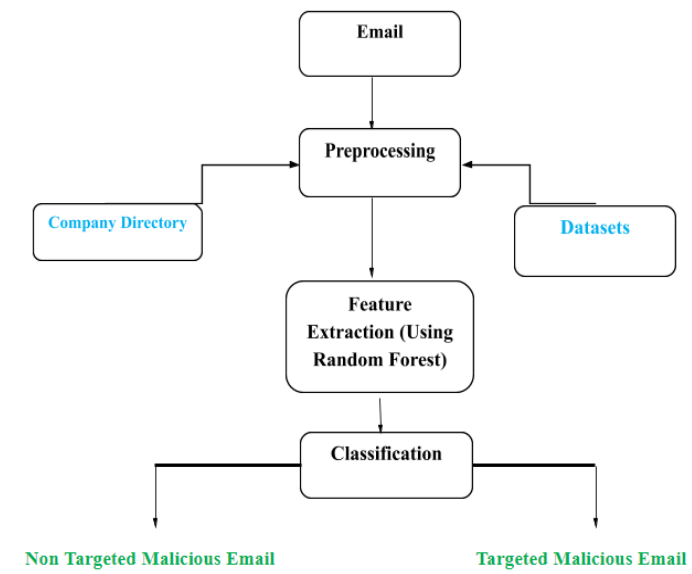

Fig 4.1: Flow Diagram

Published By:

Blue Eyes Intelligence Engineering

\& Sciences Publication 


\section{MODULE DETAILS}

\section{A. GUI Designing}

GUI stands for Graphical User Interface. It is also known as Designing part or just Presentation Logic. Utilizing the Swings idea in Java we allot zone for the measurable portrayal for the transmitted swings in one of the Technologies where we can manufacture the planning part.

We will utilize such a large number of parts like

- JPanel

- JTextBox

- JLabel and so on.

\section{B. Training Datasets}

During the preparation, a model is manufactured dependent on the attributes of every classification in a pre-arranged arrangement of email messages. The preparation dataset ought to be chosen so that it is differing in substance and subject. Each example message is marked with a particular classification. We initially perform pre-preparing to extricate tokens and decide the quantity of events of every token in every classification.

\section{Classification}

Spam separating is currently founded on computing the fluffy likeness measure between the got message and every class, for example spam or genuine. The message is then characterized by looking at its fluffy closeness measures. So as to compute fluffy comparability, we should initially decide the participation level of every token to the message . One approach to do that is by first deciding the recurrence of every token in the message. Where there is the quantity of events of token in message. Therefore, the token with the greatest number of events will be doled out an estimation of 1 , and every single other token will be alloted corresponding qualities.

\section{Analyzing sends - Resulting them}

The base for the token-classification participation, spam sifting is presently founded on ascertaining the fluffy likeness measure between the got message and every class, for example spam or genuine. The message is then arranged by looking at its fluffy comparability measures. So as to compute fluffy closeness, we should initially decide the enrollment level of every token to the message. We have to decide the recurrence of every token in the message and next enrollment degree is to be characterized. the token with the greatest number of events will be allocated an estimation of and every single other token will be relegated relative qualities.

\section{CONCLUSION \& FUTURE WORK}

In this paper we propose another email separating strategy concentrated on tireless risk and beneficiary arranged highlights that beat other accessible procedures. It helps the user to detect targeted malicious packet email for normal network. Albeit numerous casualties of ill-conceived email have cash, just certain associations have the sort of important data that yields long haul vital bit of leeway. The protocols which are used automatically predict the congestion that happens in the systematic manner and then detects the network fault in it.
For further research work, one can think about stretching out the feature of extraction to document connection metadata. Risk entertainers may incidentally leave leftovers of data, for example, document ways, time zones, or even creator names. All these highlights may relate numerous interruption endeavors into a related battle. Also, associations can follow highlights that describe the sorts and measures of email got by a specific email address.

\section{REFERENCES}

1. S. Nakamoto, "Bitcoin: A peer-to-peer electronic cash system,"2008.

2. A. Miller, A. Juels, E. Shi, B. Parno, and J. Katz, "Permacoin: Repurposing bitcoin work for data preservation," in 2014 IEEE Symposium on Security and Privacy, SP 2014, Berkeley, CA, USA May 18-21, 2014. IEEE Computer Society, 2014, pp. 475-490.

3. B. Sengupta, S. Bag, S. Ruj, and K. Sakurai, "Retricoin: Bitcoin based on compact proofs of retrievability," in Proceedings of the 17th International Conference on Distributed Computing and Networking, ser. ICDCN '16, 2016, pp. 14:1-14:10.

4. J. A. Kroll, I. C. Davey, and E. W. Felten, "The economics of bitcoin mining, or bitcoin in the presence of adversaries," Proceedings of WEIS, vol. 2013, 2013.

5. M. Rosenfeld, "Analysis of bitcoin pooled mining reward systems," CoRR, vol. abs/1112.4980, 2011

6. A. Laszka, B. Johnson, and J. Grossklags, Financial Cryptography and Data Security: FC 2015 International Workshops, BITCOIN, WAHC, and Wearable, San Juan, Puerto Rico, January 30, 2015, Revised Selected Papers. Berlin, Heidelberg: Springer Berlin Heidelberg, 2015, ch. When Bitcoin Mining Pools Run Dry, pp.63-77.

\section{AUTHORS PROFILE}

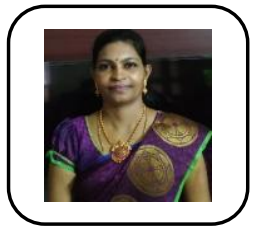

Mrs. S. Babeetha, is an Assistant professor (Sr. G) in the Department of Information Technology, SRM Institute of Science and Technology, Ramapuram Campus. She has done M.Tech in Computer Science and currently pursuing $\mathrm{PhD}$ in the same. She has 11 years of teaching experience. Her areas of research interests are in the field of Cloud computing, Machine learning, Data mining and Big data analysis.

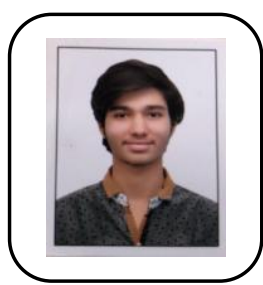

Akash Modi, is currently pursuing B.Tech in Information Technology from SRM Institute of Science and Technology, Ramapuram Campus. He has previously worked on projects based on IOT such as Waste Management System and Smart City. His areas of interests are in the field of Data Science, Cloud computing and Machine Learning.

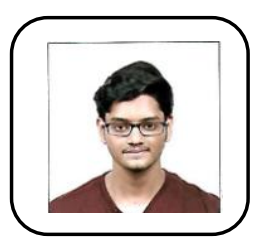

Eshwesh Gehlot, is currently pursuing B.Tech in Information Technology from SRM Institute of Science and Technology, Ramapuram Campus. He has previously worked on projects such as Smart Car and Smart stick for Blind people. His areas of interests are in the field of Networking and Cyber Security.

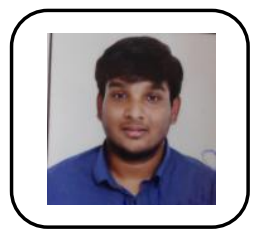

Abhishek Botikar, is currently pursuing B.Tech in Information Technology from SRM Institute of Science and Technology, Ramapuram Campus. He has previously worked on the projects such as Home automation and Traffic Management System. His areas of interests are in the field of Cloud Computing, Cryptography and Data Science.

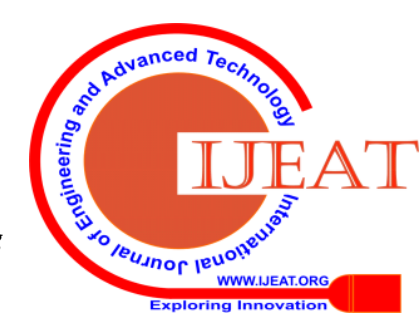

